\title{
Mechanisms for Extracting a Signal from Noise as Revealed through the Specificity and Generality of Task Training
}

\author{
Dorita H. F. Chang, ${ }^{1}$ Zoe Kourtzi, ${ }^{1,2}$ and Andrew E. Welchman ${ }^{1,2}$ \\ ${ }^{1}$ School of Psychology, University of Birmingham, Birmingham B15 2TT, United Kingdom, and ${ }^{2}$ Laboratory for Neuro- and Psychophysiology, K.U. Leuven, \\ BE-3000 Leuven, Belgium
}

Visual judgments critically depend on (1) the detection of meaningful items from cluttered backgrounds and (2) the discrimination of an item from highly similar alternatives. Learning and experience are known to facilitate these processes, but the specificity with which these processes operate is poorly understood. Here we use psychophysical measures of human participants to test learning in two types of commonly used tasks that target segmentation (signal-in-noise, or "coarse" tasks) versus the discrimination of highly similar items (feature difference, or "fine" tasks). First, we consider the processing of binocular disparity signals, examining performance on signalin-noise and feature difference tasks after a period of training on one of these tasks. Second, we consider the generality of learning between different visual features, testing performance on both task types for displays defined by disparity, motion, or orientation. We show that training on a feature difference task also improves performance on signal-in-noise tasks, but only for the same visual feature. By contrast, training on a signal-in-noise task has limited benefits for fine judgments of the same feature but supports learning that generalizes to signal-in-noise tasks for other features. These findings indicate that commonly used signal-in-noise tasks require at least three distinct components: feature representations, signal-specific selection, and a generalized process that enhances segmentation. As such, there is clear potential to harness areas of commonality (both within and between cues) to improve impaired perceptual functions.

\section{Introduction}

Breaking the camouflage of a nearby object allows a casual rambler to detect a snake in the grass, and a more experienced hiker the chance to determine whether it is a venomous European Adder or harmless grass snake. The visual processes of (1) detecting and segmenting visual elements into meaningful items, and (2) discriminating the critical features of such items are central to many everyday visual activities. Yet the functional architecture that supports these processes, and their plasticity in normal and abnormal function, is not fully understood.

Information about the depth structure of a scene (e.g., from binocular disparity) is known to assist in segmenting objects from cluttered backgrounds, as well as providing detailed object properties (e.g., shape and surface relief) that support recognition. A series of studies have examined the neuronal processing of signals related to segmentation ("coarse" tasks) as opposed to discriminative differences ("fine" tasks) using binocular disparity. This work implicates processing in visual area MT/V5 for tasks involving disparity targets in noise (e.g., DeAngelis et al., 1998; Uka and DeAngelis, 2003, 2004), whereas activity in IT and

Received Jan. 9, 2013; revised April 8, 2013; accepted May 14, 2013.

Author contributions: D.H.F.C., Z.K., and A.E.W. designed research;D.H.F.C., Z.K., and A.E.W. performed research; D.H.F.C., Z.K., and A.E.W. analyzed data; D.H.F.C., Z.K., and A.E.W. wrote the paper.

This work was supported by the European Community's Seventh Framework Programme FP7/2007-2013 (Grant agreements PITN-GA-2008-214728 and PIIF-GA-2011-299610) and the Wellcome Trust (095183/Z/10/Z).

The authors declare no competing financial interests.

This article is freely available online through the $J$ Neurosci Author Open Choice option.

Correspondence should be addressed to Dr. Andrew E. Welchman, School of Psychology, University of Birmingham, Birmingham B15 2TT, United Kingdom. E-mail: A.E.Welchman@bham.ac.uk.

DOI:10.1523/JNEUROSCI.0101-13.2013

Copyright $\odot 2013$ the authors $\quad 0270-6474 / 13 / 3310962-10 \$ 15.00 / 0$
V4 is related to perceptual judgments of fine depth differences (Uka et al., 2005; Umeda et al., 2007; Shiozaki et al., 2012). This has suggested a dissociable network, with dorsal visual areas underlying segmentation, and ventral areas subserving the processing of fine features (Roe et al., 2007).

Intriguingly, there is plasticity in the brain's signal processing for disparity segmentation: training can change the functional relevance of MT activity for signal-in-noise tasks (Chowdhury and DeAngelis, 2008). However, the link between such changes, and perceptual improvements that are known to occur in both normal (Fendick and Westheimer, 1983; Gantz et al., 2007) and stereo-deficient individuals (Nakatsuka et al., 2007; Barry, 2009; Ding and Levi, 2011), is unclear.

Here, we tested training-induced changes in behavioral performance as a means of understanding the mechanisms that support segmentation and feature discrimination. Specifically, we sought to understand how training on one type of task influences performance on another, to infer the underlying functional architecture. We start by considering the use of disparity for signal-in-noise tasks and judgments of fine feature differences (Experiment 1). Having assessed within-cue disparity learning, we then consider the extent to which learning transfers across stimulus dimensions (Experiment 2). In particular, we evaluate performance on camouflagebreaking (signal-in-noise) tasks involving motion, disparity, and orientation, and feature difference tasks (see Fig. $1 A$ ) involving these signals that are known to improve through training (e.g., Fiorentini and Berardi, 1980; Schoups et al., 1995; Liu, 1999; Folta, 2003; Saffell and Matthews, 2003).

We show that training on feature differences enhances performance on signal-in-noise tasks for the same visual property but 
A

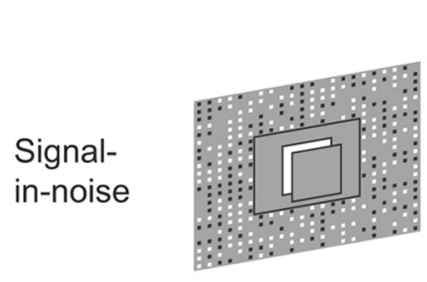

Disparity
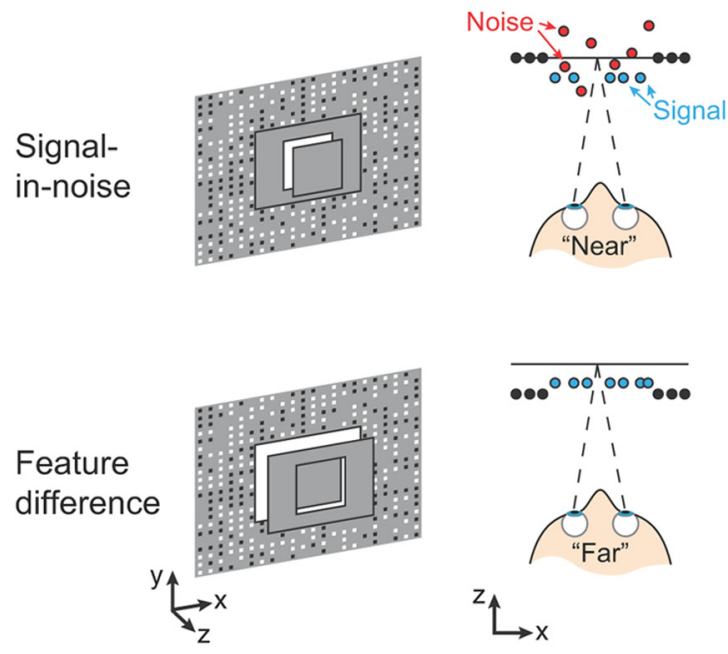

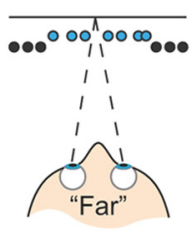

$\mathrm{z}_{\longrightarrow x}$
Motion

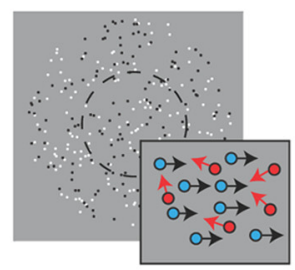

Orientation
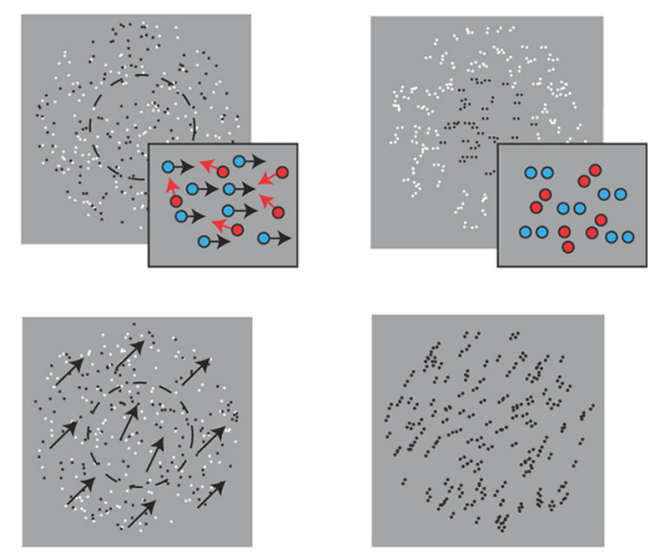

${ }^{\mathrm{y}}{ }_{\longrightarrow x}$

Pre- tests

Training

Post- tests

One task (e.g., FD depth)

(1 - 2 days)

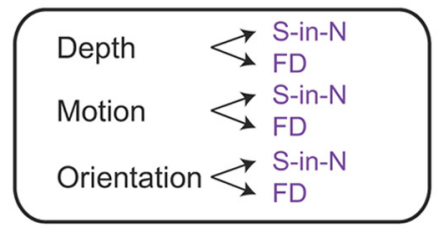

Figure 1. A, Signal-in-noise and feature difference tasks for disparity, motion, and glass pattern orientation stimuli. Disparity tasks: for the signal-in-noise task, signal dots are either near or far relative to plane of fixation; for the feature difference task, the disparity of the surround is fixed and the disparity difference between the center and the surround is varied in fine steps. Motion tasks: for the signal-in-noise task, the dots in the surround are static, whereas the dots in the central target carry a net left or right motion direction; for the feature difference task, the direction of motion conveyed by moving dots in the surround is 30 degrees clockwise or counterclockwise from vertical, and the direction of motion conveyed by the dots in the center varies finely with respect to it. Glass pattern orientation: for the signal-in-noise task, dipoles in the surround carry random orientations, whereas dipoles in the center carry a net horizontal or vertical orientation; for the feature difference task, the orientation carried by the dot dipoles in the surround is 30 degrees clockwise or counterclockwise from vertical, and orientation carried by dipoles in the center varies finely with respect to it. $\boldsymbol{B}$, The general protocol for the experiments testing on the different tasks (S-in-N, Signal-in-noise; FD, feature difference) before and after training. In Experiment 1, testing and training were restricted to the disparity tasks. In Experiment 2, testing covered all three visual cues and training took place over $2 \mathrm{~d}$.

not others (feature-specific learning). By contrast, training on signal-in-noise tasks generalizes to benefit breaking of camouflage using different visual features. These findings indicate that signal-in-noise tasks require at least three distinct components: feature representations, signal-specific selection, and a generalized process that enhances segmentation.

\section{Materials and Methods}

\section{Participants}

Participants were naive to the purpose of the study, had normal or corrected-to-normal visual acuity, were screened for stereo deficits, and provided written informed consent in line with local ethical review and approval of the work. In Experiment 1, we tested 20 observers (age 18-28 years, mean 22.9 years; 9 males): 10 participants from this group were trained on signal-in-noise depth judgments, 10 on feature difference depth judgments. In Experiment 2, we tested 28 observers (age 18-27 years, mean 22.2 years; 15 males). These participants were divided into three groups: 12 trained on motion tasks ( 6 signal-in-noise; 6 feature difference), 8 trained on depth tasks (4 signal-in-noise; 4 feature difference), and 8 trained on orientation tasks ( 4 signal-in-noise; 4 feature difference).

\section{Apparatus}

Stimuli were presented on a haploscope in which the two eyes viewed separate 20 inch Viewsonic (p225f) CRT displays through frontsilvered mirrors. The haploscope was configured for each individual so that vergence was appropriate. The viewing distance was $50 \mathrm{~cm}$, and participants used a chinrest. The stimuli were generated using MATLAB (MathWorks) with extensions from the Psychophysics Toolbox (Brainard, 1997; Pelli, 1997) and driven by a PC equipped with an nVidia graphics card configured with a spatial resolution of $1600 \times 1200$ pixels and $100 \mathrm{~Hz}$ frame rate.

\section{Stimuli}

Disparity tasks. Stimuli were random dot stereograms (RDSs) presented on a mid-gray background. The RDS depicted a $7 \times 7$ degree central target surrounded by a frame (the "surround") 14 degrees $\times 19$ degrees in size (see Fig. $1 A$, left). Dots of the RDS were randomly black or white and had a density of 2 dots $/ \mathrm{deg}^{2}$ with each dot subtending 0.15 degrees. The RDS was surrounded by a grid of black and white squares $(0.5$ degrees in size), designed to provide an unambiguous background reference and to promote stable vergence posture. Subjects were required to judge the position (in front/behind) of the central target relative to the surround. We manipulated task difficulty in one of two ways as follows: (1) We fixed the disparity of the target plane at \pm 6 arcmin (crossed and uncrossed) and varied the percentage of signal dots defining the target relative to noise dots (signal-in-noise depth task). At 100\%, all the dots in the target region had the same disparity ( 6 arcmin). Task difficulty was increased as fewer target dots were presented relative to noise dots that had a disparity chosen randomly from a uniform distribution between \pm 12 arcmin. Percentage signal corresponded to the proportion of dots that were assigned the target disparity rather than a randomly chosen disparity. Thus, a stimulus with $100 \%$ signal was maximally coherent and a stimulus with $0 \%$ signal comprised solely noise. (2) We varied the 
disparity difference between the central target and the surround (feature difference depth task). Here, the surround was assigned a disparity of 12 arcmin (crossed or uncrossed) and the disparity of the central target varied finely (1-240 arcsec) with respect to it.

Motion tasks. Motion stimuli were derived from the stochastic motion stimuli described by Newsome and Paré (1988). We generated two types of motion stimuli, both of which contained a central circular patch 7 degrees in diameter surrounded by an annulus 14 degrees in size (see Fig. $1 A$, middle). The central target and surround consisted of equal proportions of black and white dots (each 0.15 degrees in size) that were randomly positioned with a density of $2 \mathrm{dots} / \mathrm{deg}^{2}$. For the signal-in-noise motion stimulus, dots in the surrounding annulus were static, whereas dots in the central target moved with a velocity of $3.5 \mathrm{deg} / \mathrm{s}$, carrying a rightwards or leftwards motion signal. The motion signal in the central patch was varied by manipulating the percentage of dots that moved coherently in the signal direction. Thus, at $100 \%$ signal, the stimulus was coherent with all dots moving in the same direction and at $0 \%$ signal the stimulus consisted of randomly moving dots. For the feature difference motion stimulus, dots in both the surround and central target moved coherently $(100 \%)$ at the same velocity $(3.5 \mathrm{deg} / \mathrm{s})$. The direction conveyed by dots in the surround (i.e., reference direction) was on average 30 degrees clockwise or counterclockwise from vertical with a random offset of -3 to 3 degrees added to this reference direction on each trial. Task difficulty was manipulated by varying the difference in angular direction of motion in the center relative to the surround. For both signal-in-noise and feature difference stimuli, dots that moved outside of the central aperture or surround annulus were randomly repositioned on the next frame. The motion stimuli were presented on a uniform gray background.

Glass pattern orientation tasks. The glass pattern stimuli consisted of a circular central target ( 7 degrees in diameter) surrounded by a 14 degree annulus (see Fig. 1A, right). Both the central target and the annulus contained a translational glass pattern consisting of a field of random dots, each shifted by a distance of 0.25 degree in a direction $\theta$, and added to itself. The dot size was 0.15 degree and dot density was $2 \mathrm{dots} / \mathrm{deg}^{2}$. For the signal-in-noise glass pattern task, the target contained either horizontally or vertically oriented dot pairs (glass pattern dipoles). The visibility of target was manipulated by varying the proportion of signal dipoles (horizontal or vertical) relative to noise dipoles whose orientation was randomly chosen ( $0-180$ degrees). At 100\% signal, all dipoles carried the same orientation, and at $0 \%$ signal each dipole was assigned a random orientation. The color (black or white) of all the dots in the target was chosen randomly on each trial and was opposite to that for dots in the surround (i.e., providing a clear cue to the segmentation of target and surround that was also available in the disparity and motion cases). For the feature difference glass pattern stimulus, the surround and central target both contained coherently orientated dipoles. The orientation of the dipoles in the surround (i.e., reference orientation) was on average 30 degrees clockwise or counterclockwise from vertical with a random offset (in the range \pm 3 degrees) added on each trial. The difficulty of the task was manipulated by varying the difference in dipole orientations between the target and surround. Dots in the center and surround were uniformly black or white with the color chosen at random on each trial. Glass pattern stimuli were presented on a uniform gray background.

\section{Procedure}

Before starting any task, participants were provided with written instructions, printed images of the stimuli, and shown five demonstration trials to ensure they understood task requirements.

For Experiment 1, pre-training, training, and post-training tests were conducted over two consecutive sessions separated by a day. Participants completed the pre-training tests during the first session and the training and post-training tests during the second session (see Fig. 1B). For the pre-training and post-training tests, observers completed three blocks of 140 trials for each of the signal-in-noise depth and feature difference depth tasks, and a 200 trial block of a control task (see below for task details). The order of the depth tasks (signal-in-noise or feature difference) was counterbalanced, with the control task completed last in each session. During the training portion of the second session, participants were trained with six blocks (840 trials) of either the signal-in-noise depth task or the feature difference depth task. During the training phase, participants were given feedback (a high or low pitch auditory beep) on every trial answered correctly or incorrectly.

For Experiment 2, pre-training, training, and post-training tests were conducted over three sessions where sessions 2 (training) and 3 (training, post-training tests) were completed on consecutive days. The pretraining and post-training tests comprised three blocks of 140 trials for each of the signal-in-noise depth and feature difference depth discrimination tasks, three blocks of 144 trials for each of the signal-in-noise and feature difference motion tasks, and signal-in-noise and feature difference glass pattern orientation tasks. The order of the cue tested (depth, motion, glass pattern orientation) was randomized among participants. For each cue, observers completed the signal-in-noise and feature difference versions of the tasks consecutively with the order chosen at random. Training trials were completed during the second session (seven blocks corresponding to 1008 trials) and the beginning of the third session (two blocks corresponding to 288 trials). Participants were trained on one of the six tasks and tested on the other tasks before and after training (see Fig. $1 B$ ). During the training phase, participants were given feedback (a high or low pitch auditory beep) on every trial that they answered correctly or incorrectly. The precise details of training and testing for all participants were as follows. Of the 12 observers trained on the motion task, 6 were tested on only the motion and depth tasks before and after training, whereas the remaining 6 observers ( 3 trained in signal-in-noise) were tested on all six tasks. Of the 8 observers trained on the depth tasks, 2 were tested on only the depth and motion tasks, whereas the remaining 6 (3 trained in signal-in-noise) were tested on all six tasks.

Depth tasks. On each trial, observers were required to make a two alternative forced choice judgment of whether the central target was in front ("near") or behind ("far") in relation to the surround by pressing one of two keys on the keyboard. The difficulty of the task was manipulated by varying the signal of the stimulus ( $0 \%$ noise only, $20 \%, 30 \%$, $40 \%, 50 \%, 70 \%$, and $100 \%$ ) for the signal-in-noise task, or by varying the disparity difference between the target and surround $(1,6,18,24,30,60$, and $240 \mathrm{arcsec}$ ) for the feature difference task. For the feature difference task, half of the observers were tested with uncrossed disparities ("far") and the other half tested with crossed disparities ("near"). Each test block comprised 140 trials, consisting of all combinations of the 7 signal levels at uncrossed and crossed disparities, repeated 10 times (signal-in-noise task) or all combinations of the 7 disparity differences, 2 locations (in front/behind of the surround), repeated 10 times (feature difference task). The order of trial presentation was chosen at random. Stimulus duration was $200 \mathrm{~ms}$ and trials were separated by a minimum interval of $500 \mathrm{~ms}$.

Motion tasks. Observers were required to judge whether the dots in the central target carried a net leftwards or rightwards direction (signal-innoise task) or judge whether the motion direction carried by the dots in the central target was clockwise or counterclockwise with respect to the motion carried by the dots in the surround (feature difference task) by pressing one of two keys on the keyboard. On each trial, the percentage of motion signal carried by the central target (signal-in-noise task) or the difference in motion direction between the center and surround (feature difference task) was adjusted according to the QUEST staircase procedure yielding thresholds at the $82 \%$ correct level (Watson and Pelli, 1983). For the feature difference task, a maximum direction difference of 30 degrees was defined to ensure that judgments with respect to the reference were made in the same quadrant. A test block consisted of two interleaved staircases of 72 trials each comprising 4 practice trials and 68 test trials. For the practice and test trials, equal proportions of "left" and "right" trials (signal-in-noise task) or "clockwise" and "counterclockwise" trials (counterbalanced with the two reference directions) (feature difference task) were presented in random order. The initial test value of the test trials was determined as the threshold estimate from the practice trials. Stimulus duration was fixed at $200 \mathrm{~ms}$ and trials were separated by a minimum interval of $500 \mathrm{~ms}$.

Glass pattern orientation tasks. Observers were required to judge whether the stimulus carried a net vertical or horizontal signal (signalin-noise task) or whether the orientation carried by the dipoles in the 


\section{A Train on feature difference depth task}

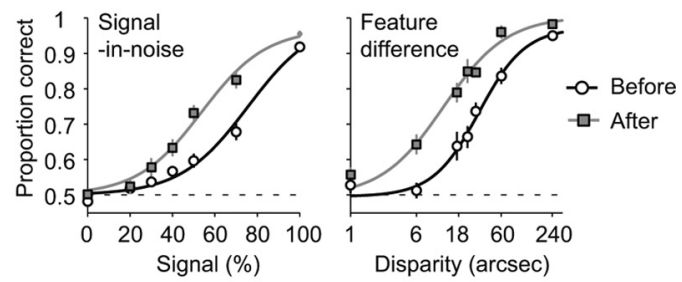

B Train on signal-in-noise depth task

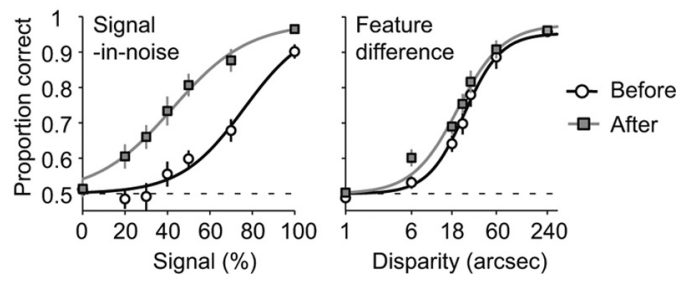

Figure 2. Psychometric functions fit to mean proportion correct judgments obtained on pre-training (black) and post-training (gray) tests for each task in Experiment 1. $A, B$, The data are shown separately for observers trained on the feature difference disparity task $(\boldsymbol{A})$ and those trained on the signal-in-noise disparity task $(\boldsymbol{B})$.

central target was clockwise or counterclockwise with respect to the orientation carried by the dipoles in the surround (feature difference task) by pressing one of two keys on the keyboard. All other task parameters and procedures were identical to those described above for the signal-innoise and feature difference motion tasks.

Control task for Experiment 1. In Experiment 1, we included a control task in the pre-training and post-training sessions to monitor participants' psychophysical performance per se. In particular, this task was used to ensure that improved performance after training was not a nonspecific effect because of increased familiarity with psychophysical testing for our naive participants. The task consisted of judging orientation (left vs right of vertical) of a large single gabor element (spatial frequency $=1.75 \mathrm{cycle} / \mathrm{deg}$; Gaussian envelope $\sigma=1.1^{\circ}$ ). As in the other tasks, stimulus duration was fixed at $200 \mathrm{~ms}$ and trials were separated by a minimum interval of $500 \mathrm{~ms}$. Performance on this task was unchanged ( $p=0.567)$ before and after training on signal-in-noise or feature difference tasks, ruling out a nonspecific training effect. Results from this condition are therefore not discussed further. This task was not included in Experiment 2, as the results from Experiment 1 indicated that differential effects were observed between conditions, making this control logically unnecessary.

\section{Data analysis}

Psychometric functions from Experiment 1 were fit using the psignifit toolbox (2.5.6) (Wichmann and Hill, 2001a, b). We used the 82\% threshold to quantify behavioral performance. Statistical analyses (repeatedmeasures MANOVAs with training group as a between-subjects factor) were conducted in SPSS (IBM).

\section{Results}

\section{Experiment 1: transfer of learning between signal-in-noise} and feature difference depth judgments

To test the role of perceptual learning in optimizing signal-innoise and feature difference depth judgments, we measured thresholds on both tasks before and after a period of training. Participants received dedicated training on either the signal-innoise depth task or the feature difference depth task (Fig. 1). We found that participants who were trained on the feature difference depth task showed improvements in both feature difference and signal-in-noise depth tasks after training. In particular, there were considerable leftward shifts in their psychometric functions when tested after training (Fig. 2A). In contrast, participants who were trained on the signal-in-noise depth task showed improvement only on the signal-in-noise task, with very little improvement observed for the feature difference disparity task (Fig. 2B).

To quantify these observations, we characterized individual participants' performance in terms of their discrimination threshold and assessed the difference in thresholds before versus after training (Fig. 3A). We analyzed these data using a repeated measures MANOVA (dependent variables: performance on each task; factor: before vs after training) with a between-subjects factor (type of training received: signal-in-noise vs feature difference). We found a significant effect of training, with thresholds significantly reduced on both the signal-in-noise task $\left(F_{(1,18)}=\right.$ $70.7, p<0.001)$ and the feature difference task $\left(F_{(1,18)}=28.4\right.$, $p<0.001)$. Performance on the signal-in-noise task improved for individuals trained on both the signal-in-noise task and the feature difference task, and while the learning effect was on average smaller for participants trained on the feature difference task (Fig. 3A, black bars), there was no statistically reliable difference in the magnitude of learning between the two training groups $\left(F_{(1,18)}=2.65, p=0.121\right)$. By contrast, improved performance on the feature difference task was markedly greater for participants training on the feature difference task $\left(F_{(1,18)}=15.7, p=\right.$ $0.001)$, and we observed no reliable improvement in the feature difference task after training on the signal-in-noise task $\left(F_{(1,18)}=\right.$ $2.6, p=0.121)$. That is, training on the feature difference task led to significant improvements on both the feature difference and signal-in-noise tasks; however, signal-in-noise task training only led to significant improvements on the signal-in-noise task. To contrast the extent of learning between training groups, we calculated a transfer index $(I)$ as follows:

$$
I=\frac{\Delta t_{\text {between }}}{\Delta t_{\text {within }}} .
$$

Here, the index is expressed as the improvement between tasks (e.g., improvement in signal-in-noise task after training on the feature difference task), $\Delta t_{\text {between }}$, relative to the improvement observed within tasks (e.g., signal-in-noise task improvement after signal-in-noise task training), $\Delta t_{\text {within }}$. A value of 0 would suggest no effect of training between tasks, and a value of 1 would indicate that training on the two tasks was equivalent in terms of post-training improvement. Bootstrapping this index suggested that feature difference task training promoted significant signalin-noise task improvement $(p<0.01)$, whereas signal-in-noise task training produced weak and nonsignificant transfer to the feature difference task $(p=0.18)$ (Fig. $3 B)$.

It is important to note that, before training, performance on both tasks was comparable for the two groups of subjects, making comparisons of the learning effects between groups uncomplicated by baseline differences. In particular, for the signal-in-noise task, the mean threshold for the group to be trained on the signalin-noise task was $81.9 \pm 3.5 \%$ versus $82.6 \pm 2.3 \%$ for the group who went on to receive training on the feature difference task $\left(t_{(18)}=-0.175, p=0.86\right)$. Similarly, for the feature difference task, performance for the participants who later received training on this task was $46.7 \pm 4.9$ arcsec versus $39.9 \pm 5.9 \operatorname{arcsec}$ for the subjects who went on to receive training on the signal-in-noise task $\left(t_{(18)}=0.87, p=0.39\right)$.

A further consideration in comparing the magnitude of training between groups relates to the mapping between the stimulus space (e.g., percentage signal) and the sensory and/or perceptual transduction of that space (e.g., percentage correct). For example, a training-induced improvement in performance from $80 \%$ 
to $70 \%$ signal does not necessarily simply equate to the same change in the perceptual process as a change from $20 \%$ to $10 \%$. We therefore considered two further data treatments to quantify the transfer of learning between signal-in-noise and feature difference tasks. First, we used variations between participants to examine the range of perceptual performance and its changes as a result of training for the two groups of participants. In particular, we fit a regression line to scatter plots of performance on the tasks before versus after training for the two groups of participants (Fig. $3 C, D$ ) and analyzed the slope term. Using this approach, a slope of unity would represent no learning effect (i.e., thresholds before training = thresholds after training). For the participants trained on the signal-in-noise task, performance on the feature difference task is close to this null result (Fig. 3D) with the data close to the $x=y$ line, and a slope parameter that is statistically indistinguishable from 1 ( $\beta=0.94 ; 95 \% \mathrm{CI}$, $0.76-1.12)$. By contrast, performance for participants trained on the feature difference task gave rise to a slope considerably lower than 1 ( $\beta=0.41 ; 95 \% \mathrm{CI}, 0.27-$ $0.56)$, demonstrating a clear learning effect. For performance on the signal-in-noise task, participants improved (slope $<1$ ) after both signal-in-noise ( $\beta=0.64 ; 95 \% \mathrm{CI}, 0.51-0.77)$ and feature difference ( $\beta=0.76 ; 95 \% \mathrm{CI}, 0.68-0.84$ ) task training, with stronger improvements for participants trained on the signal-innoise task. To quantify this asymmetry in improvements between groups, we calculated a slope-based transfer index (SI) as follows:

$$
S I=\frac{\beta_{\text {null }}-\beta_{\text {between }}}{\beta_{\text {null }}-\beta_{\text {within }}} .
$$

where $\beta_{\text {null }}$ is the slope for no learning (i.e., 1 ), $\beta_{\text {between }}$ is the slope for performance on a task when trained on the other task, and $\beta_{\text {within }}$ is the slope when training and testing where on the same task. Using this approach, we found transfer of 0.67 after feature difference task training, but only 0.10 after signal-in-noise task training, consistent with results based on threshold differences.

The second complementary analysis approach we followed was based on evaluating changes in the discriminability of the stimuli at the same stimulus intensity value before and after learning (Fine and Jacobs, 2002). In particular, after fitting the psychometric functions, we determined the stimulus intensity required for a $d^{\prime}$ of 1 (before training) and then determined the post-training $d^{\prime}$ for the same stimulus intensity. We thus quantified the learning effect as the change in $d^{\prime}$ for the same stimulus value, and computed a bootstrapped transfer index based on Equation 1, replacing stimulus intensity differences with sensitivity differences (i.e., $\Delta d^{\prime}$ ). Using this approach, we found very similar patterns of performance (Fig. $3 E$ ) as we found for the changes in thresholds, suggesting that all three data treatments resulted in a similar pattern of asymmetries between training on feature difference and signal in noise tasks.
B

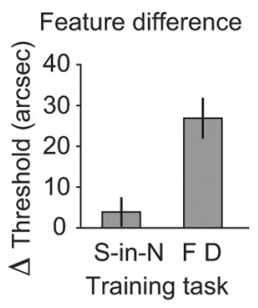

D

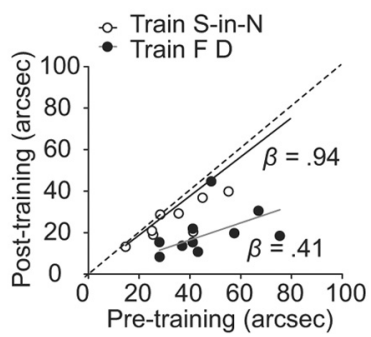

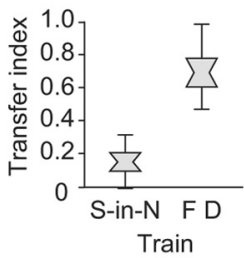

$E$

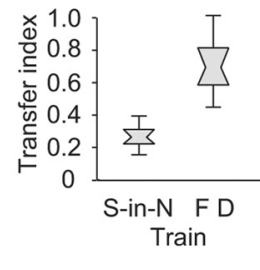

Figure 3. $A$, Data from Experiment 1 represented in terms of difference in pre-training versus post-training thresholds for each for each participant before versus after training are presented separately for tests of the signal-in-noise task and tests of the feature no learning. $E$, An alternative formulation of the transfer index computed on the basis of changes in sensitivity $\left(\Delta d^{\prime}\right)$ at the same stimulus signal level. Error bars were derived using bootstrap resampling.

One potential explanation for the minimal transfer from signal-in-noise training to feature difference task performance is that observers were not trained sufficiently on the signal-in-noise depth task. To test this idea, we recalled three observers (2-3 weeks after initial training) for additional training of 840 trials on the signal-in-noise depth task. The additional training resulted in further improvement in signal-in-noise discrimination thresholds of $10 \%$ signal on average, suggesting that learning effects last for at least a period of weeks after training. However, discrimination thresholds for the feature difference depth task changed very little $(\sim 1$ arcsec). Thus, it is unlikely that the comparatively weaker transfer of perceptual learning from signal-in-noise depth discrimination training to fine depth discriminations results from insufficient training. A further consideration in the asymmetric pattern of learning transfer is whether these effects might be the result of differences in the order of testing. This explanation is unlikely, given that we counterbalanced order across participants; nevertheless, we formally considered this possibility by including task order as a between-subject variable for our ANOVA. This indicated that the order of testing did not provide an explanation for the asymmetry: there was no main effect of task order for participants trained on the signal-in-noise task $\left(F_{(1,8)}<1, p=0.86\right)$ or those trained on the feature difference task $\left(F_{(1,8)}<1, p=0.58\right)$, nor were there any significant interaction terms involving task order. Finally, we tested the possible role of task exposure, to evaluate the amount of learning obtained on "test trials" (i.e., those without feedback in the presession and postsession). In particular, we tested whether there were systematic differences between thresholds obtained on different runs during the testing phase. We did not find any significant effect of test order (or any interactions), suggesting that the majority of learning is likely to have taken place on training trials that involved feedback. 


\section{Depth}
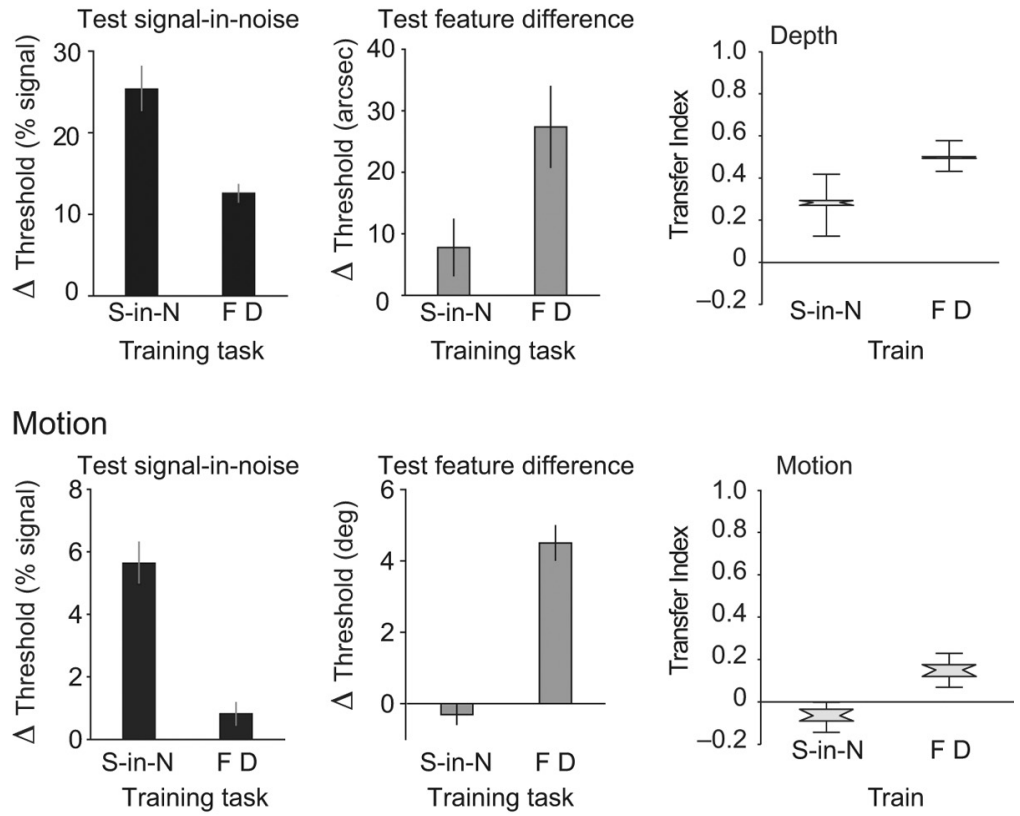

\section{Orientation}
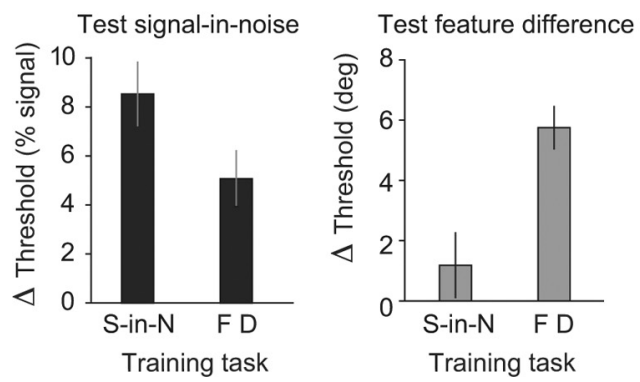

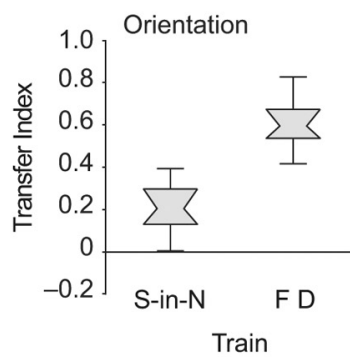

Figure 4. Differences in pre-training versus post-training thresholds for groups trained on the depth $(n=8)$, motion $(n=12)$, and orientation $(n=8)$ cues presented independently for each task to facilitate comparisons of within cues. Error bars represent \pm 1 SEM. A transfer index is presented additionally for each cue that expressed the threshold improvement between tasks relative to the threshold improvement observed within tasks. Error bars for the indices were derived from bootstrap resampling. orientation) with the order of cues and tasks randomized across participants (Fig. $1 B)$. During the training phase, individuals were trained on one of these tasks, and we thereby sought to determine the generality of learning for each task.

First, we considered the extent of transfer between signal-in-noise and feature difference tasks involving the same type of visual information. In particular, we examined the degree of learning between signal-in-noise and feature difference versions of the depth, motion, and orientation tasks. The results for the depth tasks (Fig. 4, top) were consistent with findings from Experiment 1. In particular, we found clear improvements after training for both tasks (signal-in-noise task, $F_{(1,6)}=153.9, p<0.001$; feature difference task, $\left.F_{(1,6)}=19.31, p=0.005\right)$. For the signal-in-noise task, post-training improvements were significantly greater after training on the signal-in-noise task $\left(F_{(1,6)}=19.1, p=0.005\right)$, but feature difference training still produced significant improvements $\left(t_{(3)}=10.4, p<0.001\right)$. For the feature difference task, improvements were significantly greater after feature difference task training $\left(F_{(1,6)}=6.0\right.$, $p=0.05)$, and post-training performance on this task for the signal-in-noise trained group was not significantly greater than 0 $\left(t_{(3)}=1.70, p=0.09\right)$. Thus, training on the feature difference task led to significant improvements on both tasks, whereas signal-in-noise task training improved performance on the signal-innoise task only. As for Experiment 1, we quantified learning effects by computing a transfer index (Fig. 4, right). This index confirmed that training on the feature dif-
The results from Experiment 1 suggest that the signal-in-noise and feature difference tasks rely on mechanisms with dissociable components: training on fine discriminations may enhance the trained feature (i.e., disparity) representations for both feature difference and signal-in-noise discriminations, whereas signal-in-noise task training may facilitate the segmentation of targets and the suppression of irrelevant signals but not feature representations. To test this idea further, we next investigated whether learning is specific to trained stimulus features or generalizes to stimuli defined by different image cues.

\section{Experiment 2: generalization across cues}

The results of Experiment 1 suggest that training on feature difference but not signal-in-noise discriminations provides transferable benefits to a different task involving disparity. In Experiment 2, we set out to test the generality of learning between stimuli defined by different image cues. In particular, we developed signal-in-noise and feature difference tasks that involved disparity, motion, and orientation (Fig. 1A) and tested for transfer of training across these cues. Before and after training, participants were tested on a battery of six tasks: 2 types (signal-innoise, feature difference) $\times 3$ cues (disparity, motion, ference task showed significant signal-in-noise task improvements $(p<0.01)$, whereas training on the signal-in-noise task had a comparably smaller influence on feature difference task performance, although the transfer was significant $(p<0.01)$.

Next, we considered performance on the motion tasks (Fig. 4, middle). We found significant training benefits for both tasks (signal-in-noise task, $F_{(1,10)}=71.0, p<0.001$; feature difference task, $\left.F_{(1,10)}=57.3, p<0.001\right)$. Training effects were greater for the task on which subjects were trained (signal-in-noise task, $F_{(1,10)}=34.8, p<0.001$; feature difference task, $F_{(1,10)}=34.7$, $p<0.001)$. Training on the fine feature task gave rise to a small, but significant, benefit to the signal-in-noise task $\left(t_{(5)}=2.27, p=\right.$ $0.036)$, whereas performance on the feature difference task was, on average, slightly below pre-training levels for the participants trained on the signal-in-noise task. Using the transfer indices, we observed transfer from feature difference training to signal-innoise test performance $(p<0.01)$ but no transfer from signal-innoise training to feature difference test performance $(p>0.9)$.

Finally, considering the orientation tasks (Fig. 4, bottom), we found a significant training effect for both tasks (signal-in-noise, $F_{(1,6)}=61.9, p<0.001$; feature difference, $\left.F_{(1,6)}=48.1, p=0.002\right)$, with maximal benefits on the trained tasks (signal-in-noise, $F_{(1,6)}=$ 
4.0, $p=0.093$; feature difference, $F_{(1,6)}=$ 12.7, $p=0.012$ ). Training on the feature difference task led to improved signal-innoise task performance $\left(t_{(3)}=4.43, p=\right.$ $0.011)$, whereas signal-in-noise task training did not improve feature difference task performance reliably $\left(t_{(3)}=1.10, p=0.175\right)$. Using the transfer index, we found significant transfer from feature difference training to signal-in-noise task performance $(p<0.01)$ but no transfer from signal-innoise training to feature difference task performance $(p=0.12)$.

These results demonstrate that, within each cue tested (depth, motion, orientation), there is a pattern of learning transfer between signal-in-noise and feature difference tasks that is asymmetric: training on the feature difference task promotes improvements on the signal-in-noise task. However, training on the signal-innoise task has little benefit for feature difference task performance. These findings align with findings of Experiment 1 and suggest that the asymmetric pattern of learning transfer holds across a broader range of visual feature discriminations, consistent with previous work on orientation training (Dosher and Lu, 2005).

Next, we considered the degree to which learning transfers across visual cues. Using a repeated-measures MANOVA, we compared the effects of training on the different cues (motion, depth, orientation) on performance on each task. We found that, when participants were tested on a signal-in-noise task, performance improved most for the cue on which they were trained but also improved after signal-in-noise training with other cues (Fig. 5, left). In particular, considering performance on the signal-in-noise depth task, we found that training improved performance (i.e., lower thresholds after relative to before training, $\left.F_{(1,7)}=72.6, p<0.001\right)$, and this effect was strongest for training on the disparity cue $\left(F_{(2,7)}=12.4, p=\right.$ 0.005). Thus, improvements were strongest for participants trained on the disparity signal-in-noise task, but training on the motion and orientation signal-in-noise tasks also improved performance significantly. Similarly, for the signal-in-noise motion task, there was a clear training effect $\left(F_{(1,7)}=62.6, p=0.001\right)$, with maximal benefits, on average, for participants trained on the signal-in-noise motion task (Fig. 5), although the training effect was not statistically different between groups $\left(F_{(2,7)}=3.16, p=\right.$ $0.105)$. Finally, for the signal-in-noise orientation task, performance improved after training $\left(F_{(1,7)}=174.0, p<0.001\right)$, with maximal benefits for those individuals trained on the signal-innoise orientation task $\left(F_{(2,7)}=5.54, p=0.036\right)$. Following the logic presented for the within-cue training effects, we calculated a bootstrapped transfer index that expresses the amount of transfer obtained by training between different visual features as a fraction of the amount of training obtained by training and testing on the same stimulus (Fig. 5, right). Using this index, we found significant trans-
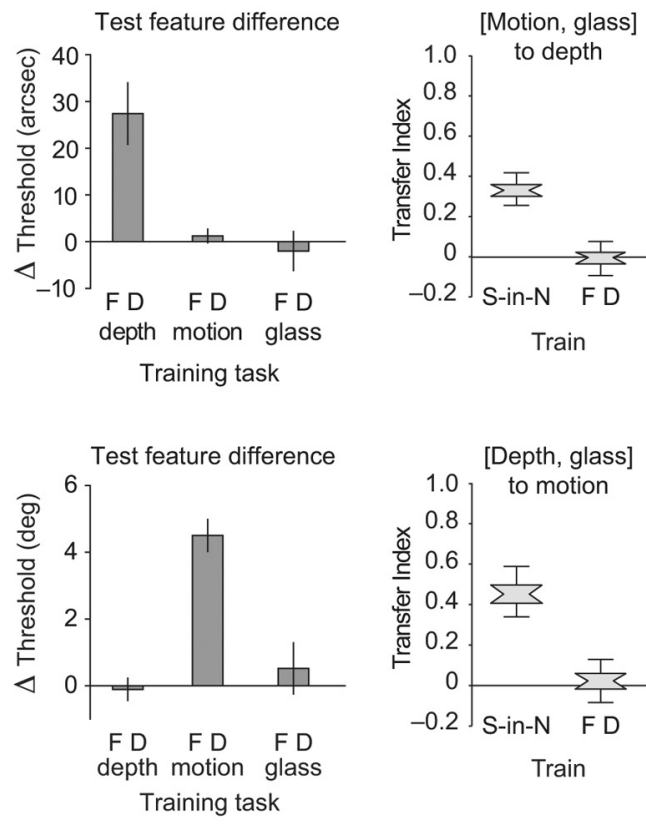

motion glass
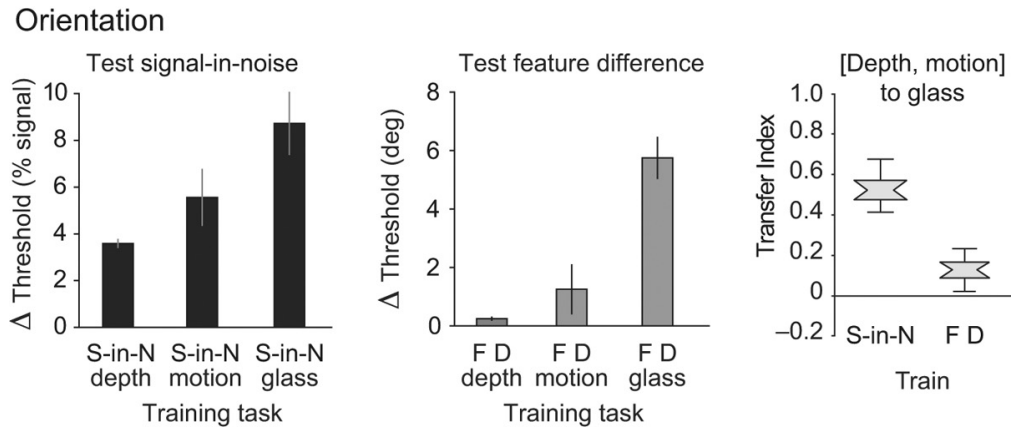

Figure 5. Differences in pre-training versus post-training thresholds for the different training groups presented independently bars for the indices were derived from bootstrap resampling.

fer between all three signal-in-noise tasks ( $p<0.01$ for all signal-innoise indices), with the extent of transfer ranging from $34-54 \%$.

In contrast to this generalization of learning across cues for signal-in-noise tasks, we found no reliable evidence of transfer between cues when participants were trained on feature difference tasks (Fig. 5, middle). Specifically, we found that, for feature difference disparity judgments, there was an improvement after training $\left(F_{(1,7)}=8.28, p=0.024\right)$ and a significant interaction with the type of training $\left(F_{(2,7)}=9.39, p=0.01\right)$. In particular, improvements were limited to participants trained on the feature difference disparity task $\left(t_{(3)}=4.17, p=0.013\right)$ and not those individuals trained on the feature difference motion $\left(t_{(5)}=0.85\right.$, $p=0.22$ ) or orientation $\left(t_{(3)}=-0.48, p=0.66\right)$ tasks. Similarly, when participants were tested on the feature difference motion task, we found a significant training effect $\left(F_{(1,7)}=21.67, p=\right.$ $0.002)$, but there were differences between training groups $\left(F_{(2,7)}=13.59, p=0.004\right)$ in that individuals trained on the feature difference motion task improved $\left(t_{(5)}=9.4, p<0.001\right)$, but not those trained on the feature difference depth $\left(t_{(3)}=-.11\right.$, $p=0.62)$ or orientation $\left(t_{(3)}=0.67, p=0.27\right)$ tasks. Finally, we found significant improvements after training for the feature difference orientation task $\left(F_{(1,7)}=33.14, p=0.001\right)$, with sig- 
Train Signal-in-Noise

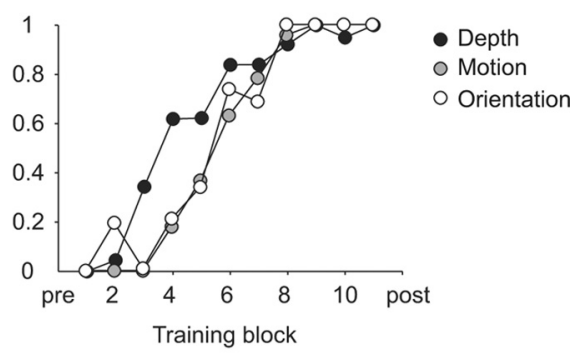

Train Feature Difference

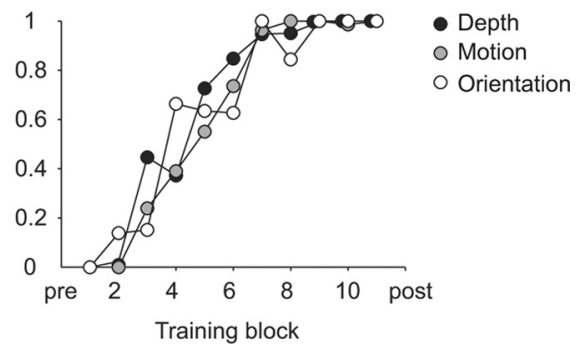

Figure 6. Group-averaged training data. Training data from each task were normalized to show the extent of improvement over training blocks as a function of the total amount learned.

nificant differences between training groups $\left(F_{(2,7)}=17.18, p=\right.$ $0.002)$. In particular, individuals trained on the feature difference orientation task improved $\left(t_{(3)}=8.2, p=0.002\right)$ but not those trained on the feature difference motion $\left(t_{(2)}=1.5, p=0.14\right)$ or depth $\left(t_{(2)}<1, p=0.36\right)$ tasks. Thus, training on fine feature tasks appears only to benefit the particular feature being trained, and there is little evidence of transfer of training effects across the different visual features of disparity, motion, and orientation. Transfer indices (Fig. 5, right) confirmed that benefits were limited to the trained stimulus feature only $(p>0.18$ for all feature difference tasks).

Although we observe a consistent pattern of results across the three different types of visual feature we have tested, the magnitude of learning might appear to be different. For instance, the change in thresholds (i.e., percentage signal) on the signal-innoise tasks are greatest for disparity and least for motion (Fig. 4). However, making such direct comparisons between tasks is not straightforward, as the percentage noise for one feature does not translate to the percentage noise for another. Therefore, to compare directly between tasks, we computed normalized learning functions that show the extent of improvement over training blocks as a function of the total amount learned (Fig. 6). In this figure, data were pooled across participants such that maximum learning reflects the total amount learned across all participants. These functions suggested comparable training effects across all the signal-in-noise and feature difference tasks. Formally, we fit individual participant training data with a single-parameter logarithmic function ( $b=k \ln (a)$, where $a$ and $b$ represent the training block and proportion of learning, respectively) to obtain estimates of learning rate $(k)$. The function was well fit to our data $(r=0.89)$ consistent with previous literature showing that performance improvement with learning is well described by an exponential model (Yang and Maunsell, 2004; Dosher and Lu, 2007). We compared the learning rate parameter between individuals in each group using a likelihood ratio test and found no significant differences between visual cues (likelihood ratio: $\chi^{2}(2$,
$N=54)=60.42, p=0.25)$ or between the two tasks (likelihood ratio: $\left.\chi^{2}(1, N=27)=38.82, p=0.07\right)$.

\section{Discussion}

In two experiments, we tested the specificity versus generalization of learning for signal-in-noise and feature difference tasks: first for stereopsis (Experiment 1) and then across cues (Experiment 2). We found that, for a given feature (e.g., disparity), training on signal-in-noise and feature difference tasks had differential benefits: feature difference task training promoted improvements for both signal-in-noise and feature difference tasks, whereas signal-in-noise training had minimal impact on feature difference tasks. By contrast, training on a signal-in-noise task benefited camouflage-breaking tasks involving other cues (e.g., signal-in-noise depth training boosted signal-in-noise motion and orientation task performance), but this between-cue facilitation did not occur for feature difference task training. This suggests that these commonly used paradigms involve dissociable mechanisms: feature difference training enhances templates that support both target detection in noise and discrimination of highly similar targets, whereas signal-in-noise training enhances a general process of target segmentation in clutter that operates independently of the cues defining the targets.

\section{Learning within image features}

First, we consider the asymmetric transfer within a given image feature. We found training on a feature difference task promotes up to $75 \%$ transfer to a signal-in-noise task, whereas transfer for the converse is limited $(<25 \%)$. This suggests that feature difference training enhances representations that are common to both tasks. Moreover, these representations are likely to contribute significantly to signal-in-noise task performance: their optimization can led to at least half the learning achieved under dedicated training on the signal-in-noise task. Critically, it is unlikely that signal-in-noise training optimizes the same representations; otherwise, we would expect transfer from the signal-in-noise task to the feature difference task.

These findings are consistent with work testing orientation judgments under conditions of high and low external noise (as our signal-in-noise and feature difference tasks, respectively). This demonstrated that low noise training transfers to both high and low noise tasks, whereas training under high noise only affects performance at high noise (Dosher and Lu, 2005). This is understood as the optimization of two independent processes: (1) filtering signals from noise and (2) internal feature representations. Performance under displays with high external noise (signal-in-noise task) requires both feature representations and noise filtering. However, noise filtering is not required for lownoise displays. Thus, signal-in-noise training helps improve noise filtering but has little benefit for noise-free viewing situations. By contrast, low-noise training optimizes internal representations that are of use for both feature difference and signal-in-noise tasks. This asymmetric transfer may reflect differences in the precision required by different tasks (Jeter et al., 2009), with more transfer expected when testing on tasks that require less precision.

\section{Generalization across image features}

Whereas we observed clear benefits of training on feature difference tasks within cues, we found a striking degree of generality in relation to performance on signal-in-noise tasks with $\sim 40 \%$ transfer between tasks involving different visual features (motion, disparity, and orientation). This indicates a significant over- 
lap in the mechanisms responsible for performance on tasks that involve extracting signals from noise.

Moreover, transfer between cues suggests that the functional architecture for signal-in-noise tasks involves (at least) three separable stages. Specifically, our data suggest that the noise-filtering processes modeled by Dosher and $\mathrm{Lu}$ (2005) comprise a processing stage that is specific to the visual feature being segmented and one that is general across cues (and potentially across sensory modalities). The logical basis for this conclusion is that transfer between signal-in-noise tasks is not complete, meaning that dedicated training on a given signal-in-noise task is likely to optimize two processes: one specific to the visual cue and the other a general noise-filtering mechanism. Based on the within-cue transfer results, we know that this specific process is unlikely to optimize feature representations themselves (otherwise, we would have observed significant benefits from signal-in-noise training to feature difference tasks). Therefore, it seems likely that signal-innoise tasks require the following: (1) feature information, (2) a selective readout of feature signals, and (3) a generalized process that extracts signals and suppresses irrelevant items. It is likely that there are significant interactions between stages 2 and 3 (for instance, in shaping readout weights), but interactions between stages 1 and 2 are likely somewhat limited in that processes that tune readout in signal-in-noise tasks have little influence on feature representations per se. It is possible that stage 3 reflects, in part, generalized processes of spatial integration. Nevertheless, as both signal-in-noise and feature difference tasks are likely to involve pooling signals across space, the extent to which spatial integration can explain the differential training effects we observe is somewhat limited.

Lu et al. (2010) implemented a Hebbian reweighting process (Petrov et al., 2005) to provide a different level of explanation for learning under high and low noise. Under their model, training in low noise promotes optimal weights for relevant features and down-weights irrelevant features. By contrast, training with noisy displays decreases the weight given to irrelevant features but cannot optimize weights for relevant features because the external noise disrupts improvements across trials. This results in asymmetric transfer between low- and high-noise tasks. Our data suggest that the process of down-weighting nonrelevant features comprises two components: one that is cue-specific and one that is general. This general process may dynamically choose among channels with high weights to select potential target signals under uncertainty caused by external noise. Training may refine this selection process, enhancing transfer between visual features but leaving performance on feature difference tasks unaffected as active selection among competing features is not needed.

It is interesting to speculate about the neural circuits involved in the different processing stages that our behavioral data suggest. Representations of visual features, such as disparity, orientation, and motion, are distributed widely in the visual cortex, suggesting multiple possible loci at which feature enhancements might occur. Neurophysiological studies of disparity and motion demonstrated a strong association between neural activity and perceptual judgments in V5/MT (Britten et al., 1992; DeAngelis et al., 1998; DeAngelis and Newsome, 1999), making this a potential locus for learning-induced changes for tasks involving these signals. Nevertheless, as neuronal responses to orientation-defined segmentation tasks in MT are not known, it is unclear whether MT responses could support the generalized signal-in-noise processes we have uncovered. Moreover, the functional relevance of activity in MT for signal-in-noise task performance changes through training (Chowdhury and DeAngelis, 2008). A more likely substrate is the parietal cortex, which is involved in readout for perceptual decisions and noise suppression. In particular, activity in the lateral intraparietal area is associated with both disparity- and motion-related perceptual decisions under manipulations of signal-to-noise (Shadlen and Newsome, 2001; Roitman and Shadlen, 2002; Gold and Shadlen, 2007; Law and Gold, 2008). Moreover, parietal cortex is implicated in attentional-control and noise (distractor) suppression (Mevorach et al., 2010; Chapman et al., 2011; Kanai et al., 2011). Thus, parietal activity may represent a generalized signal-in-noise mechanism in contrast to stimulus-specific feature readout mechanisms that may engage visual cortex.

The extent to which training effects transfer to other retinal locations and stimulus properties has been subject to considerable debate. Recent work suggests that generality is promoted by engaging tasks (Green and Bavelier, 2003, 2006a, 2006b; Li et al., 2009) and specialized protocols (Xiao et al., 2008; Zhang et al., 2010). For instance, Xiao et al. (2008) showed that specificity to retinal location, a widely observed characteristic of perceptual learning, can be abolished using a "double-training" protocol that involves feature-irrelevant training at a transfer location. This double-training protocol may promote transfer by directing spatial attention to the transfer location, which in turn reduces noise. Our experiments demonstrate a different protocol that promotes transfer across different visual dimensions. Within the framework of the reweighting model (Petrov et al., 2005), the process of dynamic selection among channels that we suggest is comparable to the attentional selection process and might be implemented in the parietal cortex described previously (Xiao et al., 2008).

In conclusion, signal-in-noise (segmentation) and fine (feature discrimination) paradigms have been used widely to study sensory processing in both human and nonhuman primates (e.g., Hawkins and Stevens, 1950; Jesteadt et al., 1977; Orban et al., 1984; Newsome and Paré, 1988). Achieving a clear understanding of the benefits of training on such tasks has implications that extend to sensory rehabilitation (Green and Bavelier, 2003, 2006a, 2006b; Li et al., 2009). Here, we show that training on feature difference tasks enhances performance on signal-in-noise tasks for the same visual feature only. By contrast, training on signal-in-noise tasks results in benefits that generalize to tasks involving other features. Our data suggest that signal-in-noise tasks involve at least three distinct components: feature representations, signal-specific selection, and a generalized process that enhances segmentation. These findings have implications not only for identifying the neural substrates of learning-dependent improvement in perceptual skills but also for developing effective rehabilitation protocols that may result in training-related improvement of different functions and generalization to everyday tasks. For instance, there is potential to target perceptual deficits (e.g., in stereoscopic vision) using paradigms that target both fine feature representations and generalized processes of noise suppression.

\section{References}

Barry SR (2009) Fixing my gaze: a scientist's journey into seeing in three dimensions. New York: Basic Books.

Brainard DH (1997) The psychophysics toolbox. Spat Vis 10:433-436. CrossRef Medline

Britten KH, Shadlen MN, Newsome WT, Movshon JA (1992) The analysis of visual motion: a comparison of neuronal and psychophysical performance. J Neurosci 12:4745-4765. Medline

Chapman CS, Gallivan JP, Culham JC, Goodale MA (2011) Mental blocks: fMRI reveals top-down modulation of early visual cortex when obstacles 
interfere with grasp planning. Neuropsychologia 49:1703-1717. CrossRef Medline

Chowdhury SA, DeAngelis GC (2008) Fine discrimination training alters the causal contribution of macaque area MT to depth perception. Neuron 60:367-377. CrossRef Medline

DeAngelis GC, Newsome WT (1999) Organization of disparity-selective neurons in macaque area MT. J Neurosci 19:1398-1415. Medline

DeAngelis GC, Cumming BG, Newsome WT (1998) Cortical area MT and the perception of stereoscopic depth. Nature 394:677-680. CrossRef Medline

Ding J, Levi DM (2011) Recovery of stereopsis through perceptual learning in human adults with abnormal binocular vision. Proc Natl Acad Sci U S A 108:E733-E741. CrossRef Medline

Dosher BA, Lu ZL (2005) Perceptual learning in clear displays optimizes perceptual expertise: learning the limiting process. Proc Natl Acad Sci U S A 102:5286-5290. CrossRef Medline

Dosher BA, Lu ZL (2007) The functional form of performance improvements in perceptual learning: learning rates and transfer. Psychol Sci 18:531-539. CrossRef Medline

Fendick M, Westheimer G (1983) Effects of practice and the separation of test targets on foveal and peripheral stereoacuity. Vision Res 23:145-150. CrossRef Medline

Fine I, Jacobs RA (2002) Comparing perceptual learning across tasks: a review. J Vis 2:190-203. CrossRef Medline

Fiorentini A, Berardi N (1980) Perceptual learning specific for orientation and spatial frequency. Nature 287:43-44. CrossRef Medline

Folta K (2003) Neural fine tuning during Vernier acuity training? Vision Res 43:1177-1185. CrossRef Medline

Gantz L, Patel SS, Chung ST, Harwerth RS (2007) Mechanisms of perceptual learning of depth discrimination in random dot stereograms. Vision Res 47:2170-2178. CrossRef Medline

Gold JI, Shadlen MN (2007) The neural basis of decision making. Annu Rev Neurosci 30:535-574. CrossRef Medline

Green CS, Bavelier D (2003) Action video game modifies visual selective attention. Nature 423:534-537. CrossRef Medline

Green CS, Bavelier D (2006a) Enumeration versus multiple object tracking: the case of action video game players. Cognition 101:217-245. CrossRef Medline

Green CS, Bavelier D (2006b) Effect of action video games on the spatial distribution of visuospatial attention. J Exp Psychol 32:1465. CrossRef Medline

Hawkins J Jr, Stevens S (1950) The masking of pure tones and of speech by white noise. J Acoust Soc Am 22:6-13. CrossRef

Jesteadt W, Wier CC, Green DM (1977) Intensity discrimination as a function of frequency and sensation level. J Acoust Soc Am 61:169-177. CrossRef Medline

Jeter PE, Dosher BA, Petrov A, Lu ZL (2009) Task precision at transfer determines specificity of perceptual learning. J Vis 9:1-13. CrossRef Medline

Kanai R, Dong MY, Bahrami B, Rees G (2011) Distractibility in daily life is reflected in the structure and function of human parietal cortex. J Neurosci 31:6620-6626. CrossRef Medline

Law CT, Gold JI (2008) Neural correlates of perceptual learning in a sensory-motor, but not a sensory, cortical area. Nat Neurosci 11:505-513. CrossRef Medline

Li R, Polat U, Makous W, Bavelier D (2009) Enhancing the contrast sensitivity function through action video game training. Nat Neurosci 12:549-551. CrossRef Medline

Liu Z (1999) Perceptual learning in motion discrimination that generalizes across motion directions. Proc Natl Acad Sci U S A 96:14085-14087. CrossRef Medline

Lu ZL, Liu J, Dosher BA (2010) Modeling mechanisms of perceptual learning with augmented Hebbian re-weighting. Vision Res 50:375-390. CrossRef Medline
Mevorach C, Hodsoll J, Allen H, Shalev L, Humphreys G (2010) Ignoring the elephant in the room: a neural circuit to downregulate salience. J Neurosci 30:6072-6079. CrossRef Medline

Nakatsuka C, Zhang B, Watanabe I, Zheng J, Bi H, Ganz L, Smith EL, Harwerth RS, Chino YM (2007) Effects of perceptual learning on local stereopsis and neuronal responses of V1 and V2 in prism-reared monkeys. J Neurophysiol 97:2612-2626. CrossRef Medline

Newsome WT, Paré EB (1988) A selective impairment of motion perception following lesions of the middle temporal visual area (MT). J Neurosci 8:2201-2211. Medline

Orban GA, Vandenbussche E, Vogels R (1984) Human orientation discrimination tested with long stimuli. Vision Res 24:121-128. CrossRef Medline

Pelli DG (1997) The VideoToolbox software for visual psychophysics: transforming numbers into movies. Spat Vis 10:437-442. CrossRef Medline

Petrov AA, Dosher BA, Lu ZL (2005) Perceptual learning through incremental channel reweighting. Psychol Rev 112:715-743. CrossRef Medline

Roe AW, Parker AJ, Born RT, DeAngelis GC (2007) Disparity channels in early vision. J Neurosci 27:11820-11831. CrossRef Medline

Roitman JD, Shadlen MN (2002) Response of neurons in the lateral intraparietal area during a combined visual discrimination reaction time task. J Neurosci 22:9475-9489. Medline

Saffell T, Matthews N (2003) Task-specific perceptual learning on speed and direction discrimination. Vision Res 43:1365-1374. CrossRef Medline

Schoups AA, Vogels R, Orban GA (1995) Human perceptual learning in identifying the oblique orientation: retinotopy, orientation specificity and monocularity. J Physiol 483:797-810. Medline

Shadlen MN, Newsome WT (2001) Neural basis of a perceptual decision in the parietal cortex (area LIP) of the rhesus monkey. J Neurophysiol 86: 1916-1936. Medline

Shiozaki HM, Tanabe S, Doi T, Fujita I (2012) Neural activity in cortical area V4 underlies fine disparity discrimination. J Neurosci 32:3830-3841. CrossRef Medline

Uka T, DeAngelis GC (2003) Contribution of middle temporal area to coarse depth discrimination: comparison of neuronal and psychophysical sensitivity. J Neurosci 23:3515-3530. Medline

Uka T, DeAngelis GC (2004) Contribution of area MT to stereoscopic depth perception: choice-related response modulations reflect task strategy. Neuron 42:297-310. CrossRef Medline

Uka T, Tanabe S, Watanabe M, Fujita I (2005) Neural correlates of fine depth discrimination in monkey inferior temporal cortex. J Neurosci 25:10796-10802. CrossRef Medline

Umeda K, Tanabe S, Fujita I (2007) Representation of stereoscopic depth based on relative disparity in macaque area V4. J Neurophysiol 98:241252. CrossRef Medline

Watson AB, Pelli DG (1983) QUEST: a Bayesian adaptive psychometric method. Percept Psychophys 33:113-120. CrossRef Medline

Wichmann FA, Hill NJ (2001a) The psychometric function: I. Fitting, sampling, and goodness of fit. Percept Psychophys 63: 1293-1313. CrossRef Medline

Wichmann FA, Hill NJ (2001b) The psychometric function: II. Bootstrapbased confidence intervals and sampling. Percept Psychophys 63:13141329. CrossRef Medline

Xiao LQ, Zhang JY, Wang R, Klein SA, Levi DM, Yu C (2008) Complete transfer of perceptual learning across retinal locations enabled by double training. Curr Biol 18:1922-1926. CrossRef Medline

Yang T, Maunsell JH (2004) The effect of perceptual learning on neuronal responses in monkey visual area V4. J Neurosci 24:1617-1626. CrossRef Medline

Zhang JY, Zhang GL, Xiao LQ, Klein SA, Levi DM, Yu C (2010) Rule-based learning explains visual perceptual learning and its specificity and transfer. J Neurosci 30:12323-12328. CrossRef Medline 